\title{
Vingt ans d'aquaculture en Afrique du Nord : évolutions, bilan critique et avenir
}

\author{
Mohamed Hichem Kara ${ }^{1, *}$, Denis Lacroix ${ }^{2}$, Sherif Sadek ${ }^{3}$, Jean Paul Blancheton ${ }^{4}$, Hélène Rey-Valette ${ }^{5}$ \\ et Mejdeddine Kraiem ${ }^{6}$ \\ 1 Laboratoire bioressources marines, ERA1, Université d'Annaba Badji Mokhtar, 23000 Annaba, Algérie \\ ${ }^{2}$ Ifremer, direction scientifique/veille et prospective, 34200 Sète, France \\ 3 Aquaculture Consultant Office, Maadi, Le Caire, Égypte \\ ${ }^{4}$ Ifremer, UMR Marbec, station de Palavas-les-Flots, 34250 Palavas-les-Flots, France \\ 5 Lameta, Université Montpellier 1, 34960 Montpellier, France \\ ${ }^{6}$ Institut national des sciences et technologies de la mer, Salambô, Tunisie
}

\begin{abstract}
Résumé - Cet article présente l'évolution de la situation de l'aquaculture en Afrique du Nord au cours des 20 dernières années, ainsi que les potentialités et contraintes spécifiques à chaque pays. Ce travail est un des produits du projet européen Aquamed (2011-2013). L'analyse des évolutions du secteur, y compris les capacités de recherche et développement, permet d'identifier les forces et les faiblesses de l'aquaculture dans les quatre pays concernés (Maroc, Algérie, Tunisie, Égypte). Cette analyse rétrospective permet aussi d'identifier des solutions pour un développement durable du secteur et la sélection de priorités d'action pour les 20 années à venir. Ce double regard, sur le passé et sur l'avenir, devrait aider les décideurs et les experts à identifier les meilleures politiques de recherche et développement dans un secteur qui a un potentiel considérable dans la région.
\end{abstract}

Mots clés : aquaculture / recherche et développement / durabilité / Méditerranée / Afrique du Nord

\begin{abstract}
Twenty years of aquaculture in North Africa: developments, critical assessment and future. This paper presents the evolution of aquaculture in North Africa during the 20 last years, and the assets and bottlenecks for each country. This work uses the results of the European project Aquamed (2011-2013). The analysis of the evolutions of this sector, including research and development allows to establish a diagnosis on the strengths and the weaknesses in the four countries (Morocco, Algeria, Tunisia and Egypt). The retrospective analysis helps in identifying solutions for a more sustainable development. It also contributes to select priorities for action in a long-term vision (2035). This double overview, on the past and the future, should help decision-makers and experts to identify the best politics for a sector, which has a high potential in the region.
\end{abstract}

Keywords: aquaculture / research and development / sustainability / Mediterranean / North Africa

\section{Introduction}

L'aquaculture en Méditerranée peut être scindée en trois catégories : la pisciculture d'eau douce et/ou saumâtre, la pisciculture marine et la conchyliculture. Depuis les années 1980, l'aquaculture marine a connu un fort développement, notamment grâce à un bon transfert des acquis de la recherche menée dans les pays intéressés par ce secteur comme la France, l'Italie ou la Grèce, vers des pays favorables à ce type d'élevage comme la Turquie, la Tunisie, Chypre, etc. L'organisation de formations a permis de répondre aux besoins

\footnotetext{
* Auteur de correspondance : kara_hichem@yahoo.com
}

des pays intéressés, puis des réseaux de professionnels ont commencé à être créés, à l'échelle nationale, en Grèce, par exemple, comme à l'échelle internationale, via la FAO et la Commission générale des pêches en Méditerranée. Le développement de la pisciculture marine s'est ensuite accéléré grâce à des capitaux extérieurs, avec une croissance forte de l'aquaculture du bar et de la daurade en Méditerranée de l'est et du sud. L'aquaculture continentale s'est rapidement développée en Égypte, où la production de tilapia, carpe et mulet principalement, est passée de $54000 \mathrm{t}$ en 1993 à 1098000 t en 2013 (GAFRD, 1997, 2014).

Hormis l'Égypte, caractérisée par une production de poisson peu cher destinée à son marché intérieur, l'aquaculture méditerranéenne produit plutôt des espèces carnassières à haute 
valeur, grossies en cages flottantes. Depuis quelques années, il existe une volonté de diversification vers de nouvelles espèces, comme le maigre (Argyrosomus regius) ou le thon (Tunnus thynnus).

La rentabilité de l'aquaculture en Méditerranée a décru depuis les années 1990-2000 pour trois raisons : la hausse continue du prix de l'aliment, la concurrence croissante des importations d'Asie, et une législation de plus en plus complexe et contraignante, dans un contexte de pression d'usage croissante sur la zone littorale (urbanisme, tourisme...). Cependant, certains pays, comme la Turquie, montrent que la négociation entre acteurs permet d'aboutir à des zonages équilibrés et à la réduction des conflits (Yucel-Gier et al., 2010). Ces exemples de planification réussie sont aussi le fruit de multiples travaux de réflexion collective menés par des organisations internationales dans le cadre de la gestion intégrée des zones côtières (IUCN, 2009).

Les dynamiques du développement de l'aquaculture en Méditerranée sont très différentes selon les pays. L'objectif du projet européen Aquamed (http:/www.aquatt.ie/aquatt-7thframework-programme/143-aquamed) était, en s'appuyant sur une plate-forme multi-acteurs (décideurs institutionnels, producteurs, chercheurs, pêcheurs, ONG...), de stimuler la coopération intra-méditerranéenne, notamment par l'identification des besoins en recherche pour un développement aquacole durable. Cet article dresse d'abord un bref historique du développement de l'aquaculture au Maroc, en Algérie, en Tunisie et en Égypte puis analyse les atouts et contraintes de chacun des pays. Enfin, les capacités de recherche et les enjeux et perspectives de développement sont évalués en utilisant une approche prospective.

\section{Historique de l'aquaculture en Afrique du Nord}

Avec $3500 \mathrm{~km}$ de façades maritimes, le Maroc est doté d'importantes ressources halieutiques. Bien qu'ayant de nombreux atouts pour l'aquaculture (secteur actif de transformation des produits de la mer, proximité des marchés européens et sites appropriés), l'aquaculture y demeure marginale (environ 500 ten 2013). Son évolution est marquée par trois phases : dans les années 1950, une dizaine de parcs produisent 300 t/an d'huitres dans les lagunes de Oualidia et de Dakhla pour le marché local. En 1986, un grand projet d'aquaculture est lancé dans la lagune de Nador, sous l'impulsion de la société Marost: huîtres et palourdes sur 1400 ha, puis bar et daurade en cage flottante. La production piscicole marine atteint $700 \mathrm{t} / \mathrm{an}$, soit $80 \%$ de la production piscicole nationale en 2005. En 2006, faute de rentabilité, l'entreprise Marost cesse son activité. La pisciculture d'eau douce (truites et carpes) a produit de manière artisanale environ 1200 t/an sur plusieurs sites en 2013, principalement dans des lacs de barrages du Moyen-Atlas.

Une relance de l'aquaculture est décidée à l'initiative du roi en 2011, avec la création de l'Agence nationale pour le développement de l'aquaculture (ANDA). Un plan global (Halieutis) est lancé en 2013, avec l'objectif de donner à ce secteur une viabilité économique en lien avec la pêche. En 2015, l'ANDA prépare un plan d'action qui prévoit une écloserie polyvalente en eau de mer pour le soutien aux projets et à la formation.
En Algérie, l'aquaculture débute en 1920 par de l'élevage extensif sur bordigue (enceinte filtrante utilisant des claies ou des filets fixes sur perche) dans la lagune Mellah. À partir des années 1980, une pisciculture d'eau douce fondée sur le peuplement d'une centaine de plans d'eau avec des espèces importées (carpes) se développe (Kara, 2012). Actuellement, une pisciculture intensive (bar et daurade en cage et en bassin) et la conchyliculture se développent. La production aquacole atteint environ 1000 t/an. La création en 2000 d'un ministère chargé de la pêche atteste de la volonté politique de promouvoir ce secteur (MPRH, 2014). L'Algérie a un fort potentiel lié à un marché local demandeur et dispose de plusieurs structures de recherche et de formation, ainsi que d'un schéma directeur de recherche et de développement à l'horizon 2025. Le cadre juridique a été adapté pour faciliter la création de concessions.

L'aquaculture en Tunisie démarre dans les années 1960 avec la conchyliculture dans la lagune de Bizerte, la carpiculture à Aïn Salem, l'élevage de tilapias dans les oasis du Sud (1970), puis de mulets dans les forages artésiens d'El Akkarit (1973) et enfin avec l'introduction, dans les lacs de barrage, de poissons d'eau douce d'Europe : carpe, gardon, rotengle, sandre, black-bass et silure (Kraiem, 1983, 1992). Ces actions sont coordonnées par l'Office national des pêches (ONP), ainsi que par différents projets financés par le gouvernement, la coopération bilatérale tuniso-française et les organismes internationaux (Kraiem, 2009). Elles permettent d'obtenir une production d'environ 1000 t/an (Losse et al., 1991). En 1977, 1'Institut national scientifique et technique d'océanographie et de pêche crée une écloserie de production de loup, daurade, sole et crevette à Ghar el Melh. Cette première station permet d'étudier et de tester les techniques de reproduction de ces espèces et de constituer les premiers stocks de géniteurs. En 1985, le Centre national d'aquaculture est construit à Monastir dans le but de maîtriser la reproduction et l'élevage des poissons marins à grande échelle et d'accompagner les promoteurs privés. À partir des années 1990, la Tunisie a accueilli les projets MEDRAP (projet régional de développement de l'aquaculture en Méditerranée)/FAO d'appui à l'aquaculture au plan régional (formation et système d'information SIPAM [Système d'information pour la promotion de l'aquaculture en Méditerranée]). En 1998, fut lancé le programme de recherche et de coopération bilatérale INSTM (Institut national des sciences et technologies de la mer)IFREMER « Aquaculture 2001 », qui a permis la restructuration et la mise à niveau des structures d'élevage expérimentales (à Monastir : aquaculture marine, à Khereddine : élevage de carnassiers, sandre, black-bass et silure dans les retenues de barrages, à Bechima, dans le Sud tunisien : pisciculture du tilapia en eaux géothermales). En 2013, en volume, l'aquaculture représente $6 \%$ de la pêche et elle continue à progresser. En eau douce, la production est d'environ $1000 \mathrm{t} /$ an (sur $14000 \mathrm{ha}$ ) avec un potentiel complémentaire de 8000 ha et une productivité en hausse $(95 \mathrm{~kg} / \mathrm{ha} / \mathrm{an})$. En conchyliculture, les meilleurs sites sont dans la lagune de Bizerte et dans le golfe de Gabès. Le potentiel de production serait de l'ordre de $5000 \mathrm{t}$, sous réserve de l'amélioration du contrôle sanitaire (présence chronique de biotoxines).

En Égypte, le tilapia du Nil et le mulet restent les principaux poissons élevés (75\% et $12,8 \%$ respectivement). La diversification porte sur la carpe $(6,6 \%)$, le poisson-chat africain, la daurade, le bar, le maigre, la sole, la crevette d'eau 
douce et de mer. Les élevages sont pratiqués en étang terrestre $(72 \%)$, en cage flottante près des embouchures des bras du Nil et en Mer Rouge (24\%), en rizière $(3,5 \%)$ et dans des réservoirs de type raceway ou bassin à fond de toile plastique, en système intensif $(0,5 \%)$ (GAFRD, 2013 ; Soliman et al., 2016). La production atteint $1098000 \mathrm{t}$ en 2013, pour une valeur de 1,35 milliard de dollars (GAFRD, 2014) et elle a été multipliée par 2,3 entre 2003 et 2012.

En 2012, l'Égypte a élaboré un cadre stratégique pour le développement économique et social de la pêche et de l'aquaculture qui va jusqu'en 2017. Le ministère de l'Agriculture considère l'aquaculture comme un puissant levier de développement, surtout dans les zones pauvres et vulnérables, avec un potentiel de 1,5 million de tonnes en 2017 (Mur, 2014). Cet accroissement de l'offre des poissons à prix modérés devrait s'accompagner d'un accès plus facile aux marchés. Le gouvernement prévoit de multiples actions : protection des sites contre la pollution, formation des gestionnaires, renfort de la recherche scientifique, adaptation de la législation, modernisation du crédit au secteur, amélioration des infrastructures, promotion de l'aquaculture intégrée dans le désert, mise aux normes des produits pour l'export, appui à l'union des coopératives aquacoles et enfin soutien au secteur de l'alimentation et de la transformation du poisson (GAFRD, 2014).

La collecte artisanale d'alevins sauvages de muges dans les lagunes côtières, le canal de Suez, les bras du Nil et les canaux de drainage a commencé dans les années 1920 sur une douzaine de sites, pour approvisionner des lacs intérieurs saumâtres, avec un prix fixe et des quotas de prélèvement (El-Zarka et Kamel, 1965). Aujourd'hui, cette collecte est possible sur 5 sites et représente en moyenne 73 millions d'alevins par an sur la période 2003-2012, dont $59 \%$ en Méditerranée et $41 \%$ en Mer Rouge (GAFRD, 2013). La forte demande a conduit à des pêches illégales, mais il n'est pas prévu d'encouragement au développement d'écloseries (Sadek et Mires, 2000).

L'exceptionnelle progression de l'aquaculture égyptienne s'explique en partie par la libération de l'initiative privée, dès lors que les règles d'usage de l'espace ont été clarifiées par l'État. L'interdiction de la pisciculture d'eau douce en cage dans le Nil en 2004, puis son autorisation partielle ultérieurement, n'ont pas cassé le dynamisme du secteur (GAFRD, 2013). À partir de 2007, les cages du Nil ont été déplacées vers l'aval des bras du Nil, sous les derniers barrages retenant l'eau douce, et donc exposés à des variations de salinité, sans apparemment de conséquences négatives sur la croissance et la survie des poissons. La production en cages atteint $250000 \mathrm{t}$ de tilapia et de mulet en 2012 (Sadek, 2013 ; Soliman et al., 2016). Le gouvernement facilite le développement de l'aquaculture d'eau saumâtre dans les terres basses du Delta. Stimulés par une demande soutenue et assurés d'une politique de long terme, les investisseurs ont multiplié les projets, préférant des élevages semi-extensifs de type artisanal aux systèmes intensifs plus coûteux en investissement et en main-d'œuvre qualifiée. L'État a aussi favorisé ce développement par l'aménagement de certaines zones, comme le triangle de Dibah dans le gouvernorat de Port-Said (Goulding et Kamel, 2013). Trois facteurs ont joué un rôle important dans cette évolution rapide : le tilapia frais coûte moins cher au consommateur que le poulet, d'où une augmentation de la demande en poisson
(+10\% par an) ; le gouvernement a favorisé l'aquaculture en 2007, avec une franchise de taxe foncière de 10 ans et un bas coût de location des terres (50 à $90 \quad \$ / \mathrm{ha})$; enfin, les producteurs ont appris à garantir des apports réguliers en produits de qualité standardisée, atout clef par rapport à des apports de pêche fluctuants (CAPMAS, 2015).

\section{Situation et contraintes}

\section{1 État des lieux}

Quel que soit le type d'aquaculture, deux classes d'entreprises se distinguent: les entreprises artisanales de petite taille, le plus souvent gérées à l'échelle familiale, dont la production annuelle est inférieure à $100 \mathrm{t}$ (type 1), et les entreprises de taille «moyenne » produisant plus de $100 \mathrm{t}$ (type 2). Le Tableau 1 montre la distribution des entreprises par type d'élevage et par pays. La situation diffère entre le Maghreb et l'Égypte. Au Maghreb, il s'agit d'une activité plutôt axée sur les espèces marines, intégrant souvent des technologies modernes. En Egypte, il s'agit surtout d'une activité à échelle artisanale et familiale (Goda, 2008). Quelques entreprises égyptiennes pratiquent des systèmes d'élevage innovants, comme la pisciculture en zone aride sur eau de forage d'eau douce et saumâtre (Sadek, 2013 ; Sadek et al., 2011).

Hormis en Tunisie, les exploitations valorisent leur production essentiellement sur les marchés nationaux. Partent à l'exportation les espèces marines les mieux valorisées : bar, daurade et maigre (GAFRD, 2013). L'Égypte peut exporter vers l'Union européene (UE) depuis 2002 et dix établissements sont agréés à cette fin, mais il manque un plan de surveillance des effluents des élevages conforme aux normes de l'UE. Ce problème est général en Afrique du Nord.

\subsection{Les contraintes et les atouts selon les pays et les types d'aquaculture}

Le Tableau 2 résume les atouts et les faiblesses de chaque pays en matière d'aquaculture. Le cas de l'Égypte est à part, en raison de l'abondance d'espaces favorables et de la forte demande locale, car la consommation de poisson est plus forte en Égypte : $19 \mathrm{~kg} /$ habitant/an contre 5 à $7 \mathrm{~kg} / \mathrm{h} / \mathrm{an}$ dans le Maghreb (FAO, 2014). La réduction du débit du Nil et l'accroissement des prélèvements d'eau par forage dans le BasDelta ont conduit à une salinisation progressive des terres basses, proches du littoral, et des grands lacs (Hereher, 2010). Entre 1990 et 2010, l'aquifère saumâtre a progressé, alors que l'aquifère d'eau douce a diminué et s'est fragmenté (Fekry, 2014). Ce phénomène a entraîné le déclin de la riziculture, mais il a créé des opportunités d'élevage pour les espèces euryhalines comme le mulet, le bar ou la daurade. Cette tendance s'est accélérée à partir des années 2000, avec les travaux d'aménagement dans le lac Manzalah et le triangle de Dibah (Sadek, 2010, 2013). Ainsi, une contrainte sur la production agricole a pu être exploitée comme une opportunité pour l'aquaculture. Il faut aussi citer le rôle pionnier de l'Égypte en matière de valorisation aquacole des eaux de forage saumâtres, parfois loin à l'intérieur des terres et souvent en zones arides, avec surtout le tilapia comme espèce produite (Sadek et al., 2011 ; Van der Heijden et al., 2013 ; Rothuis et al., 2013). 
Tableau 1. Panorama du secteur aquacole en Afrique du Nord en 2013.

Table 1. Panorama of the aquaculture sector in North Africa in 2013.

\begin{tabular}{|c|c|c|c|c|c|c|c|c|c|}
\hline \multirow[b]{3}{*}{ Échelle } & \multicolumn{8}{|c|}{ Pays } & \multirow[b]{2}{*}{ Total } \\
\hline & \multicolumn{2}{|l|}{ Maroc } & \multicolumn{2}{|c|}{ Algérie } & \multicolumn{2}{|c|}{ Tunisie } & \multicolumn{2}{|c|}{ Égypte } & \\
\hline & Nombre d'entreprises & Production & Nombre & Production & Nombre & Production & Nombre & Production & Production (tonnes) \\
\hline
\end{tabular}

Aquaculture continentale en eau douce et saumâtre (tilapia, mulet, carpe, poisson-chat...)

\begin{tabular}{|c|c|c|c|c|c|c|c|c|c|}
\hline 1 & 6 & 1235 & 1 & 10 & 19 & 1050 & ND & 1060000 & 1062000 \\
\hline 2 & & & 1 & 160 & & & & & 160 \\
\hline Total & 6 & 1235 & 2 & 170 & 19 & 1050 & ND & 1060000 & 1062000 \\
\hline
\end{tabular}

Aquaculture marine (bar, daurade, maigre, thon...)

\begin{tabular}{lllllllrr}
1 & 1 & 37 & & & & ND & 38000 & 38000 \\
2 & & & 2 & 950 & 8 & 3500 & 440 \\
Total & 1 & 37 & 2 & 950 & 8 & 3500 & ND & 38000 \\
\hline
\end{tabular}

Conchyliculture (huître, moule, palourde...)

\begin{tabular}{lrrrrrr}
1 & 17 & 100 & 20 & 3 & 500 & 620 \\
2 & 2 & 200 & & & & \\
Total & 19 & 300 & 20 & 3 & 500 & 820 \\
\hline
\end{tabular}

$\begin{array}{lrrrrrrrrr}\text { Total } & 24 & 1380 & 1 & 30 & 22 & 1550 & \text { SO } & 1098000 & 1041000 \\ 1 & 2 & 200 & 3 & 1110 & 8 & 3500 & \text { SO } & 4810 \\ 2 & 26 & 1580 & 4 & 1140 & 30 & 5050 & \text { SO } & 1098000 \\ \text { Total } & 26 & \end{array}$

ND : non déterminé ; SO : sans objet ; échelle 1 : artisanale ou familiale (moins de 100 t/an) ; échelle 2 : entreprise (plus de 100 t/an).

Source : Aquamed, 2014.

Tableau 2. Contraintes et atouts de l'aquaculture selon les secteurs et les pays d'Afrique du Nord.

Table 2. Constraints and benefits of aquaculture by sector and countries in North Africa.

\begin{tabular}{llc}
\hline \multicolumn{1}{c}{ Pays } & Maroc & Algérie \\
\hline Atouts communs & $\begin{array}{l}\text { Températures plus élevées et main-d'œuvre moins chère que sur la rive nord de la Méditerranée ; marchés locaux } \\
\text { demandeurs ; marge de progression en consommation de produits aquatiques ; proximité des marchés européens }\end{array}$ \\
\hline $\begin{array}{l}\text { Contraintes } \\
\text { communes }\end{array}$ & $\begin{array}{l}\text { Marchés peu organisés et peu transparents ; résistance des opérateurs aux normes sanitaires notamment pour l'export ; } \\
\text { bureaucratie ; coût élevé de l'aliment ; technologies modernes (écloserie et grossissement) peu connues ; manque de } \\
\text { personnel qualifié ; contraintes sur l'eau douce (quantité et qualité) ; faiblesse des systèmes de veille sanitaire et biosécurité }\end{array}$ \\
\hline
\end{tabular}

Atouts (A) et contraintes (C) par milieu d'élevage et par pays

$\begin{array}{llll}\text { Pisciculture } & \text { A. Secteur de pêche puissant ; A. Demande locale forte } & \text { A. Longue expérience } \\ \text { marine } & \text { bon potentiel de marché local } & \text { C. Secteur de pêche peu dans ce secteur dont } \\ \text { développé ; peu de sites } & \begin{array}{l}\text { des réussites ; volonté } \\ \text { de l'État en R\&D }\end{array} \\ & \begin{array}{l}\text { expériences plutôt } \\ \text { négatives }\end{array} & \begin{array}{l}\text { C. Manque } \\ \text { d'investisseurs }\end{array}\end{array}$

\begin{tabular}{|c|c|c|c|c|}
\hline $\begin{array}{l}\text { Pisciculture } \\
\text { continentale }\end{array}$ & $\begin{array}{l}\text { C. Marché réduit pour le } \\
\text { poisson d'eau douce; peu de } \\
\text { sites }\end{array}$ & $\begin{array}{l}\text { A. Des sites encore mal } \\
\text { valorisés } \\
\text { C. Poisson peu apprécié ; } \\
\text { risque de manque d'eau }\end{array}$ & $\begin{array}{l}\text { C. Peu de sites ; risque } \\
\text { de pénurie d'eau sur } \\
\text { certains sites }\end{array}$ & $\begin{array}{l}\text { A. Disponibilité d'espaces ; } \\
\text { investisseurs nombreux } \\
\text { C. Peu de planification et } \\
\text { de contrôle; pollutions et } \\
\text { risques d'eutrophisation }\end{array}$ \\
\hline Conchyliculture & $\begin{array}{l}\text { C. Peu de naissain ; risques } \\
\text { d'efflorescences (blooms) } \\
\text { toxiques; produits peu valorisés }\end{array}$ & $\begin{array}{l}\text { C. Marché peu } \\
\text { développé ; peu de sites }\end{array}$ & $\begin{array}{l}\text { A. Marchés à l'export } \\
\text { C. Risques de toxines }\end{array}$ & $\begin{array}{l}\text { C. Marché réduit ; peu de } \\
\text { sites ; risques de pollution } \\
\text { chronique ; prix élevés }\end{array}$ \\
\hline
\end{tabular}


Tableau 3. Indicateurs des efforts de recherche et de développement en aquaculture en Afrique du Nord.

Table 3. Aquaculture research and development efforts indicators in North Africa.

\begin{tabular}{|c|c|c|c|c|c|}
\hline Paramètre & \multicolumn{5}{|c|}{ Pays } \\
\hline Nombre d'institutions de R\&D concernées & 7 & 12 & 12 & 7 & 38 \\
\hline Nombre de doctorants et post-doctorants & 44 & 33 & 87 & 100 & 264 \\
\hline Total des chercheurs, doctorants et post-doctorants & 77 & 76 & 157 & 350 & 660 \\
\hline Production (en millier de tonnes - année 2012) & 1,58 & 1,14 & 5,05 & 1038 & 1046 \\
\hline$\%$ production nationale/production régionale & 0,3 & 0,2 & 0,5 & 99 & 100 \\
\hline Rapport production/chercheur (t/pers) & 20 & 15 & 32 & 3000 & - \\
\hline
\end{tabular}

Source : Aquamed, 2014.

La Tunisie, malgré peu de sites favorables, a développé en 20 ans un secteur aquacole performant, principalement grâce à un bon soutien de la recherche et à des entrepreneurs déterminés. Ces exemples montrent que, si les pays d'Afrique du Nord n'ont pas les mêmes atouts naturels que d'autres pays méditerranéens comme la Grèce ou la Turquie, il est possible d'y développer une filière aquacole complète créant des emplois et de la richesse. Dans les 4 pays, plusieurs sites pourraient être mieux valorisés (golfe de Gabès, de Tunis et d'Hammamet, lagunes de Mellah, Nador et Dakhla, côte de M'diq), mais les experts déplorent deux défauts récurrents majeurs : une administration trop bureaucratique pour être efficace dans l'accompagnement des projets et un manque de techniciens locaux compétents. Au-delà des avantages comparatifs et des plans de développement détaillés, c'est la capacité à créer un développement intégré de l'ensemble des composantes de la filière aquacole qui fait le succès ou l'échec d'une filière aquacole (Da Silva et al., 2005).

Le Maroc affiche une politique volontariste, mais ne tire pas profit des sous-produits d'une importante industrie de la pêche et d'un marché local diversifié. La création de l'agence de développement ANDA et les études en cours devraient permettre de donner un cadre solide au redémarrage du secteur.

En Algérie, l'aquaculture n'a pas connu le développement escompté, malgré la volonté politique et des atouts réels, notamment en termes d'avantages financiers et fiscaux. Le développement espéré semble dépendre d'abord de la capacité à mettre en place une organisation en cohérence avec des objectifs réalistes. Il faut souligner l'effort actuel de restructuration de l'ensemble de la filière, y compris en matière de formation et de recherche finalisée (MPRH, 2014).

En Tunisie, l'aquaculture commence à acquérir une masse critique suffisante pour attirer les investisseurs (FAO, 2014). Le pays dispose d'une vraie expérience en aquaculture (Kraiem, 1992) et d'un potentiel de sites aménageables. La place de l'aquaculture d'eau douce reste modeste, mais pourrait se développer. L'apparition de fermes de grossissement de thon constitue une nouvelle filière attractive, mais elle reste fragile car dépendante des captures de pêche. Ce pays possède un important gisement de palourdes (Ruditapes decussatus) et une capacité d'épuration aux normes internationales (Medhioub et al., 2013). Cette filière représente des centaines d'emplois potentiels dans les gouvernorats du Sud et des marchés à l'exportation (Italie et Espagne).

En Égypte, en 1994, les analyses du réseau SIPAM de la FAO prévoyaient une progression lente des productions avec des méthodes simples, sur des sites connus, et pour des espèces traditionnelles. Or, en 20 ans, ce pays a décuplé sa production avec des méthodes variées, sur de nouveaux sites et avec de nouvelles espèces. Malgré un contexte difficile, ce secteur fait preuve d'une vitalité et d'une réactivité exceptionnelles, et l'Égypte couvre aujourd'hui $20 \%$ de ses besoins en protéines animales par l'aquaculture (GAFRD, 2014). Les déterminants majeurs de cette réussite sont la démographie, la demande alimentaire et les prix. Cependant, la vision prospective et les outils de planification qui ont été transmis (projets de l'UE : Perseus, Pegaso, Medina, Force...) sont aujourd'hui peu utilisés dans les cercles de décision. Un développement mal maîtrisé du secteur risque de faire émerger de sérieux problèmes : pathologies, conflits avec la pêche traditionnelle ou l'agriculture, surtout dans une perspective de changement climatique et de salinisation des eaux dans le delta du Nil où est pompée l'eau pour l'irrigation.

\section{Capacités de recherche, enjeux et perspectives}

\subsection{Recensement des capacités de recherche et d'accompagnement}

D’une manière générale, les pays d'Afrique du Nord disposent de nombreuses structures de recherche spécialisées en aquaculture. Au Maroc, 2 départements d'université, 2 instituts et le Centre national de l'hydrologie et de la pisciculture d'eau douce. En Algérie, 4 départements d'université et 2 instituts. En Tunisie, 2 départements d'université et 4 instituts. En Égypte, 10 universités, 2 facultés, 2 instituts, un laboratoire central et une organisation internationale. Dans tous les cas, on observe un déficit de coordination entre les acteurs de l'aquaculture et une relative faiblesse de la formation technique et du suivi des producteurs. Le Tableau 3 montre les efforts de recherche et de formation dans le secteur. L'Égypte est dans une situation paradoxale, avec un faible niveau apparent de soutien de la recherche au développement, malgré 10 associations 
Tableau 4. Indicateurs des publications scientifiques en aquaculture en Afrique du Nord (1993-2014).

Table 4. Aquaculture scientific publications indicators in North Africa (1993-2014).

\begin{tabular}{lllll}
\hline Paramètre & Maroc & Algérie & Tunisie & Égypte \\
\hline Nombre de publications (total de 345) & 45 & 21 & 124 & 166 \\
Taux de co-publications (\%) & 73 & 71 & 78 & 59 \\
$1^{\text {er }}$ pays partenaire (\% des co-publications) & Portugal (33\%) & France (36 \%) & France $(22 \%)$ & États-Unis (10 \%) \\
Nombre de pays partenaires & 13 & 8 & 26 & 36 \\
Domaines de publication majeurs & Général / huîtres / algues & Algues & Poissons marins & Poissons d'eau douce \\
\hline
\end{tabular}

d'aquaculture qui dépendent de la GAFRD (General Authority for Fish Resources Development), et 5 associations liées au ministère des Affaires sociales (EgAS, 2014). Le WorldFish Centre, organisme international de recherche dédié à la réduction de la pauvreté et de la faim par la pêche et l'aquaculture, y est actif via un réseau de partenaires directs comme des communautés de pêcheurs ou d'éleveurs dans des situations de vulnérabilité alimentaire liées à des catastrophes naturelles ou des déplacements forcés (WorldFish, 2014).

Plusieurs indicateurs issus des enquêtes d'Aquamed permettent d'évaluer la capacité d'accompagnement de la recherche par rapport à la taille du secteur et à ses performances. Ils sont présentés dans le Tableau 3 et montrent des différences nettes entre les pays du Maghreb et l'Égypte : la Tunisie présente un fort potentiel relatif de recherche, contrairement à l'Égypte.

\subsection{Types de recherche}

Pour chacun des pays, les projets de recherche ont été caractérisés en fonction des thématiques abordées. Au Maroc, une grande variété de sujets est abordée mais seuls quatre thèmes atteignent ou dépassent $10 \%$ de fréquence : les cycles biologiques, l'élevage larvaire, la nutrition et l'environnement. En Algérie, peu de thèmes sont étudiés en dehors des cycles biologiques, de l'environnement et de la qualité des produits. En Tunisie, on observe une très grande diversité des thèmes abordés, mais aucun thème n'est supérieur à $12 \% \mathrm{du}$ total. Les quatre thèmes les plus fréquents sont: la reproduction, la nutrition, l'environnement et la diversification des espèces élevées. En Égypte, les domaines de recherche sont diversifiés et concernent en particulier la gestion des étangs, la reproduction et l'élevage de nouvelles espèces (poisson-chat africain, carpes chinoises), l'amélioration génétique du tilapia (croissance, survie, tolérance au froid et à la salinité, résistance aux maladies), le développement de nouveaux aliments, l'optimisation d'élevages intégrés dans les rizières, la pathologie, et la transformation des poissons. Des études économiques sont aussi menées pour identifier les voies d'amélioration de la rentabilité des exploitations familiales.

Sur le long terme, on observe une stabilité des thèmes de recherche avec des efforts portant plutôt sur la structuration des équipes que sur l'inflexion de thèmes de recherche. Ainsi, en Tunisie, le programme Aquaculture 2001 a permis de fédérer les nombreux projets de recherche de l'INSTM en 10 axes structurants. Une démarche similaire a été engagée en Algérie en 2012 (Ferlin et al., 2014 ; MPRH, 2014).
L'analyse transversale des thèmes d'étude montre que, si les sciences biologiques sont assez bien représentées, il apparaît un net déficit en socioéconomie, optimisation des systèmes de production et valorisation pour les marchés. Or, les programmes de recherche des grands pays aquacoles européens montrent un renforcement des recherches dans ces domaines (European Marine Board, 2014 ; NSTC, 2014). De plus, les besoins d'intégration de toutes les activités marines dans une vision de moyen terme, prenant en compte les principes de durabilité, ne sont pas couverts, bien qu'identifiés dans ces quatre pays (IUCN, 2009 ; Ecorys, 2012).

\subsection{Approche par la bibliométrie}

Une étude bibliométrique a été menée dans le Web of Science sur les publications en aquaculture des pays d'Afrique du Nord, dans la période 1993-2014 (Salaün, 2014). Le Tableau 4 donne des indicateurs de cette production scientifique et montre un fort taux d'ouverture au partenariat international dans tous les pays.

Le domaine d'étude majeur est l'aquaculture d'eau douce et saumâtre (126 publications) en raison du poids important de ce secteur pour l'Égypte (96 publications). Le poisson marin arrive en second rang avec 69 publications. Viennent ensuite l'aquaculture générale, puis les mollusques, algues et crevettes. La dynamique du nombre de publications par pays (Fig. 1) montre un taux à peu près stable depuis 2004 pour l'Algérie et le Maroc et un accroissement marqué pour l'Égypte et la Tunisie, qui multiplient par 3 leur production scientifique entre 2005 et 2014. Cependant, la quantité de publications reste faible en regard du nombre de chercheurs.

\subsection{Approche par la prospective}

Des exercices de prospective ont été menés en collaboration avec de nombreux acteurs de l'aquaculture en Égypte, en Algérie et au Maroc dans le cadre de projets européens. Des ateliers de prospective, tels celui du Caire (UE-Pegaso en 2013), d'Algérie (jumelage UE-Algérie en 2014) et du Maroc (jumelage UE-Maroc en 2015), ont ainsi contribué à éclairer les stratégies de recherche des organismes participants et à transmettre un outil de réflexion collective opérationnel.

D'une manière globale, trois facteurs devraient renforcer le développement de l'aquaculture en Afrique du Nord: la démographie qui prévoit entre 20 à 25 millions d'habitants supplémentaires sous 15 ans (CIHEAM-Plan Bleu, 2009), une consommation croissante de produits aquatiques en raison de leur rôle reconnu en santé humaine (Cohen et al., 2005), et 


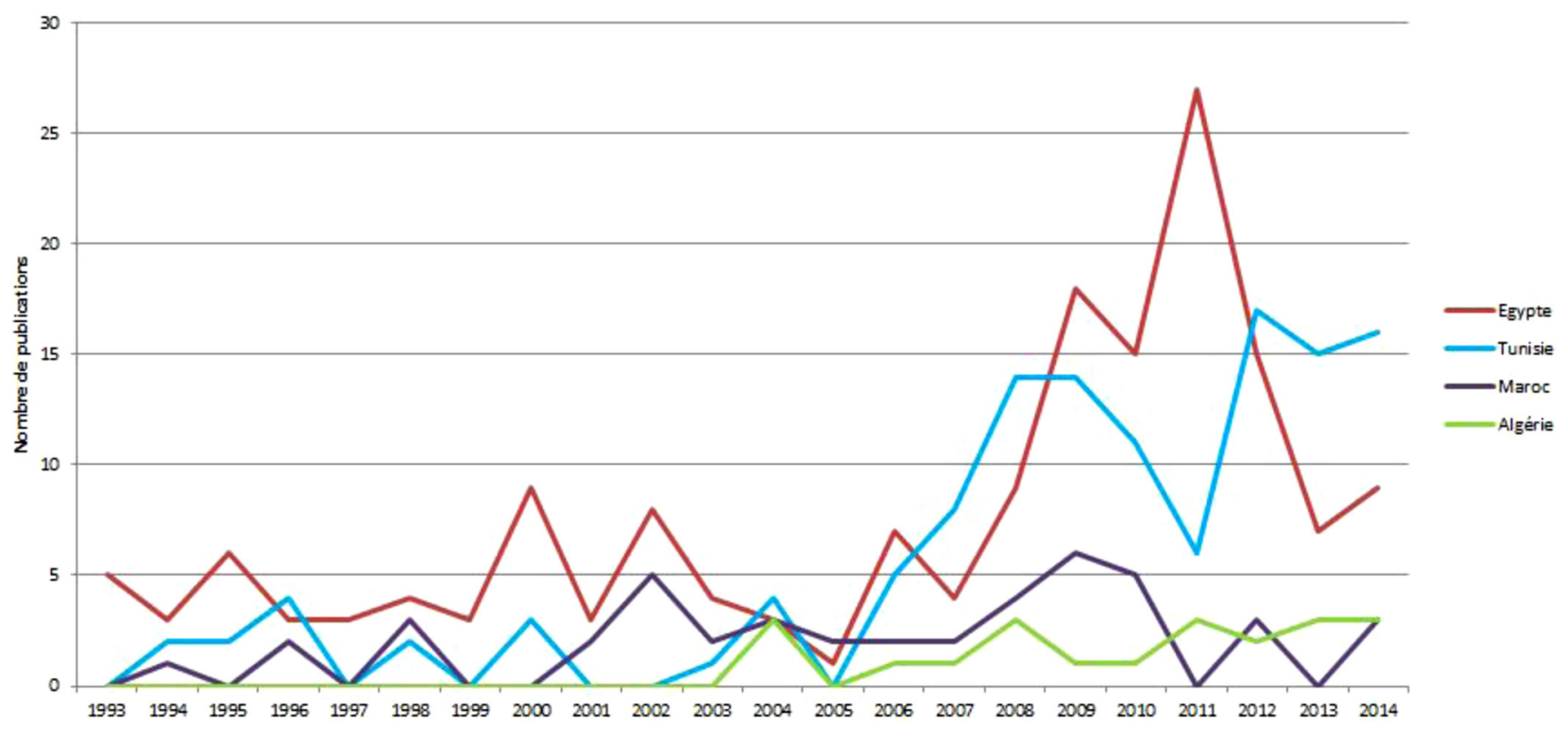

Fig. 1. Évolution du nombre de publications scientifiques en aquaculture par pays (source : Salaün, 2014).

Fig. 1. Evolution of aquaculture scientific publications by country.

enfin l'accroissement des sites disponibles pour l'aquaculture, avec la salinisation inexorable des zones basses et le potentiel offert par les lacs et barrages et les eaux de forage en zone désertique.

Dans le domaine marin, les études dites « intégrées », s'appuient encore trop souvent sur la juxtaposition de disciplines, alors que les enjeux devraient être traités de manière transversale. De plus, une approche spécialisée ne facilite pas la découverte des synergies possibles, comme dans les projets d'installation d'éoliennes marines (Buck et al., 2010) ou pour l'amélioration de la résilience des villes côtières et des deltas face au changement climatique (Smith et al., 2011 ; Van der Voorn et al., 2011). En aquaculture, comme dans bien d'autres domaines, les décideurs doivent disposer d'une capacité d'anticipation des effets du changement global, afin d'optimiser les adaptations. Une approche prospective faciliterait l'émergence d'une aquaculture durable intégrant les enjeux de long terme et prenant en compte les contraintes communes majeures que sont la démographie, la pénurie d'eau douce, la montée du niveau de la mer et les attentes sociétales.

\section{Conclusion et perspectives}

L'analyse de 20 années de recherche et de développement en aquaculture en Afrique du Nord permet de tirer deux enseignements majeurs : le développement du secteur est toujours lié à la conjonction de conditions favorables précises et connues (marché, environnement, systèmes d'élevage, espèces) et à une masse critique de décideurs réactifs. L'exemple le plus net est celui de l'Égypte, où l'apparition de conditions favorables a permis de décupler l'aquaculture en 20 ans. Par ailleurs, quand un cadre d'accueil est préparé avec une vision de long terme en impliquant les principaux acteurs, les projets bénéficient d'un contexte a priori favorable, ce qui réduit les risques de blocage, facilite les démarches et accélère la mise en œuvre. Pour cela, la plate-forme multi-acteurs méditerranéenne, mise en place dans le cadre du projet Aquamed, et les plates-formes nationales et régionales « miroir » sont particulièrement utiles (Aquamed, 2014). Ces structures «miroir » sont constituées comme Aquamed par un réseau associant tous les acteurs de l'aquaculture : producteurs, institutionnels et chercheurs. La dynamique créée est ensuite à entretenir en adaptant la recherche aux problèmes qui apparaissent. C'est clair en Égypte, où la vitesse du développement doit rester maîtrisée sous peine de dysfonctionnements environnementaux et sociétaux majeurs. La coopération internationale et la formation se révèlent d'autant plus efficaces qu'elles s'ancrent dans la durée, comme l'a montré la succession de programmes de coopération bilatérale entre la Tunisie et la France depuis 1997. Dans un monde en réseaux et connecté à de multiples banques de données, la valeur ajoutée de tout projet revient à la qualité du travail sur le terrain, à l'intégration des connaissances dans un dialogue entre tous les acteurs, à la capitalisation des expériences, à l'anticipation des crises, et enfin à la capacité d'éclairer les choix stratégiques par l'approche prospective (Ecorys, 2012). Les pays d'Afrique du Nord ont accès maintenant, et collectivement, à ce potentiel. Il dépend d'eux de le valoriser, en s'appuyant sur l'expérience acquise et la volonté de construire collectivement une aquaculture durable.

Remerciements. Les auteurs tiennent à remercier tous les experts qui ont contribué à la relecture critique de cet article et à leurs multiples commentaires constructifs : F. Breton (UAB/ Pegaso) ; P. Ferlin (CGAAER) ; R. Flos (UPC/CSIC) ; A. Lane (EAS); D. Sauzade (Plan Bleu); F. René (CGPM/ CAQ) ; F. Simard (UICN/Écosystèmes marins); Y. Zaky (bibliothèque d'Alexandrie). 


\section{Références}

Buck BH, Krause G, Michler-Cieluch T. 2010. Meeting the quest for spatial efficiency: progress and prospects of extensive aquaculture within offshore wind farms. Helg. Mar. Res. 62(3): 269-281.

CAPMAS. 2015. Website of the Central Agency for Public Mobilization and Statistics. Available from www.capmas.gov.eg.

Aquamed. 2014. The future of research on aquaculture in the Mediterranean region. Available from http://www.aquatt.ie/aquatt7th-framework-programme/143-aquamed.

CIHEAM-Plan Bleu. 2009. Repenser le développement rural en Méditerranée. Méditerra 2009, Sciences Po/Les presses, 371 p.

Cohen JT, Bellinger DC, Connor WE, Kris-Etherton PM, Lawrence RS, Savitz DA, et al. 2005. A quantitative risk-benefit analysis of changes in population fish consumption. Am J Prev Med 29: 325-334.

Da Silva NJR, Beuret JE, Mikolasek O, Fontenelle G, Dabbadie L, Espagnoli MI. 2005. Dynamiques de développement de la pisciculture et politiques publiques dans la vallée du Ribeira, État de Sao Paulo (Brésil). Cah Agric 14: 59-63.

Ecorys. 2012. Blue growth: scenarios and drivers for sustainable growth from the oceans, seas and coasts. Study on mature, emerging and pre-development economic activities at sea in 2020. In: Final report for DG Mare (UE), $202 \mathrm{p}$.

EgAS. 2014. The activity of the Egyptian Aquaculture Society from 2001 to 2014 in the development of the Egyptian aquaculture sector. EgAS, in Arabic language, $7 \mathrm{p}$.

El-Zarka S, Kamel F. 1965. Mullet fry transplantation and its contribution to the fishery of the inland brackish lakes in Egypt, U A R Proc Con Fish Counc Mediterr 8: 209-226.

European Marine Board. 2014. Navigating the future IV. In: Position paper No. 20 of the European Marine Board (Ostend, Belgium), $204 \mathrm{p}$.

FAO. 2014. La situation mondiale des pêches et de l'aquaculture. Rome: FAO, 255 p.

Fekry AM. 2014. An overview of groundwater in Egypt. Preparation for a knowledge Development and Exchange Community (KDEC) on Integrated Agriculture - Aquaculture (IAA) with brackish water worshop. 30 November, 2014. Cairo, Egypt: Wageningen University \& the Egyptian Ministry of Agriculture, $31 \mathrm{p}$.

Ferlin P. 2014. Rapport final de jumelage DZ11AAAG07 sur la mission de coopération UE-Algérie sur le renforcement des capacités du CNRDPA. Afonso E, Baroiller JF, Carpi P, Ferlin P, Forest A, Grati F, Guary JC, Lacroix D, Lericolais G, Ponis E, Poussard P. 53 p.

GAFRD. 1997. Statistics of fish production of year 1996. General authority of fisheries research and development. Ministry of Agriculture and Land Reclamation, $112 \mathrm{p}$.

GAFRD. 2013. Statistics of fish production of year 2012. General authority of fisheries research and development. Ministry of Agriculture and Land Reclamation, $106 \mathrm{p}$.

GAFRD. 2014. Statistics of fish production of year 2013. General authority of fisheries research and development. Ministry of Agriculture and Land Reclamation, $106 \mathrm{p}$.

Goda AMA. 2008. Small-scale fish culture: guiding models of aquaponics and net-enclosures fish farming in Egypt. Egypt J Aquat Res 34: 320-337.

Goulding I, Kamel M. 2013. Institutional, policy and regulatory framework for sustainable development of the Egyptian aquaculture sector. In: World fish, project report: 2013-39, 31 p.

Hereher M. 2010. Vulnerability of the Nile Delta to sea level rise: an assessment using remote Sensing. Geomat Nat Hazards Risk 1: $315-321$.
IUCN. 2009. Site selection and site management in Mediterranean aquaculture; guide for the sustainable development of Mediterranean aquaculture. Part 3, $301 \mathrm{p}$.

Kara MH. 2012. Freshwater fish diversity in Algeria with emphasis on alien species. Eur J Wildl Res 58: 243-253.

Kraiem MM. 1983. Les poissons d'eau douce de Tunisie : inventaire commenté et répartition géographique. Bull Inst Nat Sci Tech Oceanogr Pêch Salambô 10: 107-124.

Kraiem MM. 1992. Révision de l'inventaire des poissons d'eau douce de Tunisie. Bull Soc Sci Nat Tunis 20: 98-100

Kraiem MM. 2009. La recherche aquacole à l'INSTM : résultats et perspectives (1998-2008). In: Rapport INSTM, 21 p.

Losse GF, Nau W, Winter M. 1991. Le développement de la pêche en eau douce dans le Nord de la Tunisie. In: Coop. Tech. Tunisoallemande (CGP/GTZ), $417 \mathrm{p}$.

Medhioub W, Amzil Z, Soudant P, Hamza A, Medhioub N, Kraiem M. 2013. Étude de l'impact de l'alimentation sur la détoxification de la palourde Ruditapes decussatus contaminée in situ par les gymnodimines (Boughrara, Tunisie). Bull Inst Nat Sci Tech Mer Salambô 40: 73.

MPRH. 2014. Bilan 2012-2014, prospective 2030 et projet aquapêche 2020. Ministère des Pêches et des Ressources halieutiques d'Algérie, $70 \mathrm{p}$.

Mur R. 2014. Development of the aquaculture value chain in Egypt. In: Report of the National Innovation Platform Workshop, Cairo, 19-20 February 2014. Cairo: WorldFish, 39 p.

NSTC. 2014. National strategic plan for federal aquaculture research 2014-2019. National Science and Technology Council; Committee on Science; Inter-agency Working Group on Aquaculture, $21 \mathrm{p}$.

Rothuis A, Pieter van Duijn A, Roem A, Ouwehand A, Piji W, Rurangwa E. 2013. Aquaculture business opportunities in Egypt. In: Wageningen URC. LEI Report 2013-039, Imares report C091/13.

Sadek S, Mires M. 2000. Capture of wild finfish fry in Mediterranean coastal areas and possible impact on aquaculture development and marine genetic resources. Isr J Aquac Bamidgeh 52: 77-88.

Sadek S. 2010. Egyptian marine shrimp farming: problems, challenges and prospects for future development. In: Atti del convegno " $\mathrm{La}$ risorsa Crostacei nel Mediterraneo: ricerca, produzione e mercato", organized by CONARGA and Veneto Agricoltura, pp. 27-36.

Sadek S. 2013. Aquaculture site selection and carrying capacity estimates for inland and coastal aquaculture in the Arab Republic of Egypt. In: Ross LG, Telfer TC, Falconer L, Soto D, AguilarManjarrez J, eds. Site selection and carrying capacities for inland and coastal aquaculture. FAO/IoA, University of Stirling, Expert Workshop, 6-8 Dec. 2010. UK. FAO Fisheries and Aquaculture Proceedings No. 21, pp. 183-196.

Sadek S, Frige T, Sabry M, Asfoor M, Bolos S, El-Smadony II, et al. 2011. Egyptian desert aquaculture status of the art. Mag Eur Aquac Soc Aquac Eur 36: 5-11.

Salaün A. 2014. Aquaculture des pays du Nord de l'Afrique 1993-2014. Données bibliométriques. Bibliothèque La Pérouse, $33 \mathrm{p}$.

Smith TF, Daffara P, O'Toole K, Matthews J, Thomsen DC, Inayatullah S, et al. 2011. A method for building community resilience to climate change in emerging coastal cities. Futures 43 (7): 673-679. DOI: 10.1016/j.futures.2011.05.008.

Soliman NF, Dalia M, Yacout M. 2016. Aquaculture in Egypt: status, constraints and potential. Aquacult Int 24: 1201-1227. doi: 10.1007/s10499-016-9989-9.

Van der Heijden P, Bloom G, Sadek S, Elsamadony E, Eweas M, ElDib H, et al. 2013. Development of integrated aquaculture agriculture systems with brackish and salt water, Egypt. In: Projects BO-10-001-223 and BO-10-011-132: sustainable 
aquaculture in brackish and salt water. Wageningen University \& Research Centre, Holland Centre for Development Innovation (CDI), $64 \mathrm{p}$.

Van der Voorn T, Pahl-Wostl C, Quist J. 2011. Combining backcasting and adaptive management for climate adaptation in coastal region: a methodology and a South-African case study. Futures 44(4): 346-364. DOI: 10.1016/j.futures.2011.11.003.
WorldFish. 2014. Investment in Egypt's aquaculture sector to increase food security and prvide employment. Disponible sur http://www. worldfishcenter.org/our-research/ongoing-projects/investment-inegypts-aquaculture\#.VF5LoGB0zIU.

Yucel-Gier G, Arisoy Y, Pazi I. 2010. A spatial analysis of fish farming in the context of ICZM in the Bay of Izmir-Turkey. Coast Manag 38: $399-411$.

Citation de l'article : Kara M.H, Lacroix D, Sadek S, Blancheton JP, Rey-Valette H, Kraiem M. 2016. Vingt ans d'aquaculture en Afrique du Nord : évolutions, bilan critique et avenir. Cah. Agric. 25: 64004. 